SCAN-9610008

$$
\text { sin } 9641
$$

\title{
Characteristics of charged particle multiplicities distribution in relativistic heavy-ion interactions
}

\author{
P. L. Jain and G. Singh \\ High Energy Experimental Laboratory \\ Department of Physics \\ State University of New York at Buffalo \\ Buffalo, New York 14260
}

July 1,1996

\begin{abstract}
Multiplicity and pseudorapidity distributions of shower particles produced in interactions of the ${ }^{197} \mathrm{Au}$ at $10.6 \mathrm{~A}$ $\mathrm{GeV},{ }^{28} \mathrm{Si}$ at $14.5 \mathrm{~A} \mathrm{GeV},{ }^{16} \mathrm{O}$ and ${ }^{32} \mathrm{~S}$ at $200 \mathrm{~A} \mathrm{GeV}$ beams were studied in nuclear emulsion. The pseudorapidity $(\eta)$ distributions are approximately Gaussian in shape. Their widths increase monotonically from central to peripheral collisions with energy and with the size of the target. The widths of the pseudorapidity distributions, the values of their centroid, pseudorapidity density and $\eta_{\text {peak }}$ values are compared among the different groups of their multiplicity at different energies as a function of centrality for all four beams.
\end{abstract}

PACS numbers: $25.70 . \mathrm{Np}, 12.38 . \mathrm{Mh}$

\section{INTRODUCTION}

Relativistic heavy-ion physics aims to probe previously experimentally inaccessible regions of high energy and baryon density of nuclear matter. Much of the motivation arises from the production of a new state in which the individual quarks and gluons are deconfined: quark-gluon plasma [1]. Recent lattice QCD calculations [2] predict a critical temperature $T \approx 200 \mathrm{MeV}$ corresponding to an energy density of the order of $\approx 3$ $\mathrm{GeV} /(\mathrm{fm})^{3}$ which is essential for QGP formation. Another related issue is the degree of "stopping" of the incident projectile hadrons. Previous studies have shown that these conditions are created mostly in central collisions. Some insight into the dynamics of such collisions can be gained by the investigation of the multiplicity and pseudorapidity density distributions of charged particles $[3,4]$. We compare the results on such studies for
${ }^{16} \mathrm{O}$ and ${ }^{32} \mathrm{~S}$ at $200 \mathrm{~A} \mathrm{GeV},{ }^{28} \mathrm{Si}$ at $14.5 \mathrm{~A} \mathrm{GeV}$ and ${ }^{197} \mathrm{Au}$ at $10.6 \mathrm{~A} \mathrm{GeV}$ interactions with emulsion target. Experimental details were discussed in previous publications [3-7] and thus we only highlight the main points for the experimental technique here.

Within the last few years a large amount of experimental work in high energy heavy-ion physics has been done with electronic detectors, which generally have limited coverage in the pseudorapidity $(\eta)$ range $[8,9]$. On the other hand emulsion is a global detector and has full $4 \pi$ coverage. It acts as a target as well as a detector in which the angle of emittance of about $0.1 \mathrm{mrad}$ can be easily achieved. Thus with minimal-bias data set, one can make exclusive observation in multiplicity and pseudorapidity distributions of all the charged particles produced in individual interactions with different projectiles at various energies. For any systematic study on shower particles in an event as a function of centrality, it is also desirable to identify the charges of the projectile fragments (PFs) observed in the interaction for the complete analysis on an event as was done in Ref. [5].

\section{EXPERIMENTAL DETAILS}

We are using the ${ }^{197} \mathrm{Au}$ at $10.6 \mathrm{~A} \mathrm{GeV}$ (BNL Expt. No. 875 ), ${ }^{28} \mathrm{Si}$ at $14.5 \mathrm{~A} \mathrm{GeV}$ (BNL Expt. No. 847), ${ }^{16} \mathrm{O}$ and ${ }^{32} \mathrm{~S}$ at $200 \mathrm{~A} \mathrm{GeV}$ (CERN Expt. No. EMU08) beams. The details of the data acquisition procedure and angle measurement technique are given in Refs. [6] for the ${ }^{197} \mathrm{Au}$ and ${ }^{28} \mathrm{Si}$ beams and for the ${ }^{16} \mathrm{O}$ and ${ }^{32} \mathrm{~S}$ beams in Ref. [7] . For each beam, scanning was done using the along-the-track technique and thus we collected about $1400,1300,1000$ and 900 events for the ${ }^{197} \mathrm{Au}$ 
(beam $A$ ) at $10.6 \mathrm{~A} \mathrm{GeV},{ }^{32} \mathrm{~S}$ (beam B) at $200 \mathrm{~A} \mathrm{GeV}$. ${ }^{28} \mathrm{Si}$ (beam $\mathrm{C}$ ) at $14.5 \mathrm{~A} \mathrm{GeV}$ and ${ }^{16} \mathrm{O}$ (beam D) at $200 \mathrm{~A} \mathrm{GeV}$, respectively. From the minimum-bias (MB) sample of each beam, we excluded the electromagnetic and the elastic events [10]. In the case of the ${ }^{197} \mathrm{Au}_{u}$ beam $A$, we put further restrictions and selected those events which has PFs of charge $1 \leq Z \leq 17$. These restrictions on the ${ }^{197} \mathrm{Au}$ beam reduced the numbers of events to $275,379,811,345$ for beams $A, B, C$ and $D$, respectively. The emulsion detector is quite suitable for recording all the charged particles: PFs of $Z>1[5,11]$, singly charged shower ( $N_{s}$ produced) particles mostly pions with a mixture of kaons, protons with $\beta>0.7$ and ionization $I<1.4 I_{0}$ ( $I_{0}$ being the minimum ionization) in the very forward direction; target associated grey particles mostly knock-out protons $\left(N_{g}\right)$ plus low energy pions with $0.3<\beta<0.7$ or $1.4 I_{0}<I<5 I_{0}$ with energy between $40-400 \mathrm{MeV}$ and the evaporation fragments from the target, the black particles $\left(N_{b}\right)$ with $\beta<0.2$ or $I>5 I_{0}$ and with energy $<40 \mathrm{MeV}$. Thus the total target associated particles $N_{h}=N_{b}+N_{g}$. The spectator PFs of charge $Z=1$ (proton $N_{p}$ ) are mostly produced within a narrow forward cone of $\theta<\theta_{c}=0.2 / P_{\max }$. For the number of shower particles $\left(N_{s}\right)$ produced in an interaction, all of the spectator protons are excluded from the number $N_{s}$. The counter $[8,9]$ experiments generally do not make distinction between particles with $\beta>0.7$ and the $0.3<\beta \leq 0.7$ and thus they generally include the grey particles with shower particles. So for any comparison of emulsion data with the data accumulated with the counter techniques, one must be aware of these facts.

\section{RESULTS AND DISCUS- SION}

\section{(a) Angular distribution of shower particles}

The angles of all the shower particles were determined with the relative primary method [3]. In these measurements we have excluded the electron pair tracks from pion-gamma conversion $\left(\gamma \rightarrow e^{+} e^{-}\right)$. The electron tracks generally stay together and have a considerable amount of Coulomb scattering with very small opening angles between them [12]. The emission angle $\theta$ of the track gives pseudorapidity $\left(\eta_{a b}=-\ln \tan (\theta / 2)\right)$. For the beams $B, C$ and $D$, the pseudorapidity distributions for the MB data as well as for the central events were presented in Refs. $[4,7,11]$. The distributions for these beams fit very well with Monte Carlo code VENUS rather than FRITIOF $[3,7]$. So first we present the pseudorapidity distribution for only central events for the ${ }^{197} \mathrm{Au}$ beam $A$ and for that we used a different sample of 240 central events with $N_{s}>50$ where the charges of the PFs were $Z \leq 20$. These events are divided into three different groups with multiplicities: $N_{s} \leq 125,125<N_{s} \leq 225$ and $N_{s}>225$. The pseudorapidity density distribution of these events is shown in Fig. 1(a) and they are fitted with a Gaussian distribution. The highest density observed with shower particles between $0.1 \leq \eta \leq 4.0$ is with $\left\langle N_{s}\right\rangle \approx 300$ and $N_{P F}=0$. For the highest multiplicity events the peak value of the normalized density as shown in Fig. $1(a)$ is $\rho_{\text {max }}=136 \pm 22, \eta_{\text {peak }}=2.12 \pm 0.29$ and $\sigma=0.93 \pm 0.10$. The excess of particles beyond $\eta=4.0$ is due to the spectator (PF) protons. There is a depletion of particles in the very forward direction which is a consequence of energy conservation.

(b) Comparison of different parameters observed from $\mathrm{MB}$ data sample of ${ }^{32} \mathrm{~S},{ }^{28} \mathrm{Si}$ and ${ }^{16} \mathrm{O}$ with ${ }^{197} \mathrm{Au}$ beam

The number of $M B$ events for beams $A, B, C$ and $D$ are $275,379,811$ and 345 , respectively. From the angles of their shower particles, the pseudorapidity density distribution for beams is plotted and is shown in Fig. 1(b). For these four beams with different projectiles at different energies, one sees evidence for limiting fragmentation in the target fragmentation region, where the distributions from four energies fall on top of each other for $\eta<1.0$. The peak of the distribution $d N_{s} / d \eta$ is clearly shifted towards smaller $\eta$ values with increasing centrality. The backward displacement is accompanied by a slight reduction of the width of the $\eta$ distribution, which is expected as the degree of stopping increases. The data for each beam was distributed into six different multiplicity sets so that each set within a particular beam has a reasonable number of events. For each set of events in a particular beam, we plotted the pseudorapidity distributions which were fitted with Gaussian distributions with different $\sigma_{0}$ values for each beam. Then for each set of shower particles in a particular group we determine the values of the parameters $\left\langle N_{s}\right\rangle, \sigma_{0},\left\langle\eta_{l a b}\right\rangle$ (centroid), $\eta_{\text {peak }},\left(d N_{s} / d \eta\right)_{\text {peak }}$, $\left\langle N_{p}\right\rangle,\left\langle N_{g}\right\rangle$ and $\left\langle N_{\text {part }}\right\rangle$. We are interested in knowing how these parameters varies with mass, energy and centrality.

One of the important parameters that one needs in heavy-ion interactions is to measure the centrality of the collision. In electronic experiments, it is quite common to use $E_{t}$ or $P_{t}$ as an energy or transverse momentum transfer parameter as a measure of the impact param- 
eter. In emulsion works, some authors have used the multiplicity $\left(N_{B}\right)$ to determine the centrality of the event [13]. It is not a good parameter especially when the projectile is heavy like ${ }^{197} \mathrm{Au}$ or ${ }^{208} \mathrm{~Pb}$ nuclei. We find that either $N_{p}$ or $Z_{\text {bound }}$ is a good indicator about the impact parameter [5], where $N_{p}=Z_{\text {beam }}-Z_{\text {bound }}$ and $Z_{\text {bound }}=\sum_{Z_{i}>1} Z_{i} . N_{p}$ and $Z_{\text {bound }}$ are complementary to one another. In this process one has to determine very carefully the charges of all the PFs within a reasonable errrors which is a tedious task in emulsion work. We find that $N_{p}$ is good parameter as shown in Refs. [5,11]. For a simple geometrical picture of collisions, we have shown earlier [11] a monotonic relationship between the number of projectile proton $N_{p}$ and the impact parameter $b$ through $\bar{b}=b\left(N_{p}\right) / b_{\max }=\left[\int_{N_{p}}^{\infty} d P_{N_{p}}\right]^{1 / 2}$, where $d P_{N_{p}}$ is the normalized probability distribution for the measured proton multiplicity and $b_{\max }$ is the maximum impact parameter.

(i) $\left\langle\eta_{\text {lab }}>\right.$ vs $<N_{p}>/ Z_{b e a m}$

In Fig. 2(a) is plotted the centroid $\left\langle\eta_{l a b}\right\rangle$ of the Gausian fits of the $\eta$ distribution as a function of centrality (i.e. $\left\langle\eta_{l a b}\right\rangle$ vs $\left\langle N_{p}\right\rangle / Z_{b e a m}$ ). The errors shown here are all statistical in nature, throughout this paper. The centroid of the low energy ${ }^{197} \mathrm{Au}$ beam is almost constant and independent of centrality; for the ${ }^{28} \mathrm{Si}$ beam, the distribution is also constant but for large $\left\langle N_{p}\right\rangle$ the constant values decrease. For the ${ }^{16} \mathrm{O}$ and ${ }^{32} \mathrm{~S}$ beams, both lighter projectiles at higher energies, show similar behaviors as shown by the ${ }^{197} \mathrm{Au}$ and ${ }^{28} \mathrm{Si}$ beams, but their centriod values are much larger as compared to the ${ }^{197} \mathrm{Au}$ and ${ }^{28} \mathrm{Si}$ beams; the energy effect. The same kind of observations were made in Refs. [8,9,14]. The backward shift of the peak position with increasing centrality is due to the fact that the ratio of target participants to projectile participants increases as the impact parameter decreases when the projectile is smaller than the target. This is also clear from Fig. 1(b). We may mention that in emulsion the heavier element is $(\mathrm{AgBr})$ which is smaller than ${ }^{197} \mathrm{Au}$ nuclei.

(ii) $\sigma_{\mathrm{o}}$ vs $<N_{p}>/ Z_{\text {beam }}$

In Fig. 2(b) we show the width $\left(\sigma_{0}\right)$ of the Gaussian fits to the data which decreases with the centrality ( $\left\langle N_{p}\right\rangle / Z_{\text {beam }}$ ) but it increases significantly with the increase in the projectile energy at $200 \mathrm{~A} \mathrm{GeV}$. The behavior of the ${ }^{32} \mathrm{~S}$ and ${ }^{16} \mathrm{O}$ beams at $200 \mathrm{~A} \mathrm{GeV}$ are similar at the same energy. On the other hand, the low energy beams, ${ }^{197} \mathrm{Au}$ and ${ }^{28} \mathrm{Si}$, display low $\sigma_{0}$ values and show similar behavior upto $\left\langle N_{p}\right\rangle / Z_{\text {beam }} \approx 0.6$ and after that they behave slightly differently where the projectile mass plays an important part. At the last two points the heavier beam ${ }^{197} \mathrm{Au}$ has narrow $\sigma_{0}$ values than for the ${ }^{28} \mathrm{Si}$ beam. Previously we have proved [15] that when we go from a lighter target $(\mathrm{H}, \mathrm{CNO})$ to a heavier target $(\mathrm{Ag}, \mathrm{Br})$, then the width $\sigma_{0}$ is affected. A heavy target has more spectator matter than a small target. The fragmentation of the target gives the largest contribution in the backward regions and widens the pseudorapidity distribution. If we take a high energy beam of ${ }^{16} \mathrm{O}$ or ${ }^{32} \mathrm{~S}$ at $200 \mathrm{~A} \mathrm{GeV}$, there is a substantial breakup of the target and projectile into nucleons even for peripheral collisions. This means that in peripheral collisions a larger fraction of the charged particles stems from the fragmentation of target and projectile as compared to central collisions. The backward and forward regions are dominated by target and projectile fragmentation, respectively, whereas the region around midrapidity is dominated by the produced particles. Since the yieid of charged particles in the backward and forward regions does not increase as strongly with centrality as in the central region the width decreases with centrality. Thus the width obtained from the experimental data increases with the size of the target and decreases with increasing centrality. The same trend was observed in Refs. $[8,9,14]$.

(iii) $\eta_{p e a k}$ vs $<N_{p}>/ Z_{b e a m}$

Fig. 2(c) displays the pseudorapidity $(\eta)$ of the centroid, i.e. $\eta_{\text {peak }}$ position values as a function of centrality. The values for the ${ }^{197}$ Au beam (for larger mass) are slightly higher than for the ${ }^{28} \mathrm{Si}$ beam, both at low energy and these values decrease with centrality. High energy beams ${ }^{32} \mathrm{~S}$ and ${ }^{16} \mathrm{O}$ have similar values with centrality but their peak $\eta$ position values are quite large as compared to the low energy ${ }^{197} \mathrm{Au}$ and ${ }^{28} \mathrm{Si}$ beams. It coincides with the observations made in Ref. [8].

(iv) $\left\langle N_{s}>\right.$ vs $\left\langle N_{p}\right\rangle / Z_{\text {beam }}$

In the geometrical model, when two nuclei collide, the nuclei in the overlap regions known as the "participants" collide with each other, thereby slowing down and creating new particles whereas the projectile and target remnants outside the overlap volume known as the "spectators" continue to move at nearly projectile and target rapidity. The number of participants and spectators is determined by the collision geometry. Thus the relative sizes of the projectile and the target are important and this is observed in Fig. 2(d) where we plot the average shower particle multiplicity $\left\langle N_{s}\right\rangle$ vs $\left\langle N_{p}\right\rangle / Z_{\text {beam }}$. The linearity of $\left\langle N_{\mathrm{s}}\right\rangle$ for light projectiles ${ }^{16} \mathrm{O},{ }^{28} \mathrm{Si}$ 
and ${ }^{32} \mathrm{~S}$ beams are upto $\left\langle N_{p}\right\rangle / Z_{\text {beam }} \approx 0.9$ and for the heavy ${ }^{197} \mathrm{Au}$ beam it is only up to 0.60 and after that the multiplicity values increase much more rapidly in the ${ }^{197} \mathrm{Au}$ beam as compared to the ${ }^{16} \mathrm{O},{ }^{28} \mathrm{Si}$ and ${ }^{32} \mathrm{~S}$ beams. These distributions do not show linearity over the whole range while $N_{p}$ distribution is linear over the whole impact parameter range [11]. One can see why multiplicity should not be used as an indicator of the centrality as it was done in Ref. [13].

(v) $<N_{\text {part }}>$ vs $<N_{p}>/ Z_{\text {beam }}$

The number of participating nucleons $\left\langle N_{\text {part }}\right\rangle$ in heavy-ion collisions is an important parameter which can be estimated by $N_{\text {part }}=Z_{\text {beam }}-\sum_{Z_{i}>1} Z_{i P F}-$ $N_{\text {proj.sp }}$, where $N_{\text {proj.sp }}$ represents projectile spectator ( $Z=1)$ among the shower particles and are produced in the very forward direction as was discussed earlier in section 2. The number of target spectator grey particles, mostly knock-out protons $\left(N_{g}\right)$, are very easy to recognize in emulsion as compared to electronic detectors. Fig. 3(a) exhibits the distribution of projectile participants $<N_{\text {part }}>$ as a function of centrality. The number $N_{\text {part }}$ increases almost linearly with $\left\langle N_{p}\right\rangle / Z_{\text {beam }}$ for all beams. For smaller projectile $\left({ }^{16} \mathrm{O},{ }^{28} \mathrm{Si}\right.$ and $\left.{ }^{32} \mathrm{~S}\right)$ the $\left\langle N_{p}\right\rangle / Z_{\text {beam }}$ increase is very small while for the heavy projectile ${ }^{197} \mathrm{Au}$ beam, the $\left\langle N_{p}\right\rangle / Z_{\text {beam }}$ increase is very large. Collision geometry is playing very important part here.

(vi) $\left\langle N_{g}>\right.$ vs $<N_{p}>/ Z_{\text {beam }}$

In Fig. 3(b) we show the dependence of the number of target participants on centrality. The supperposition model assumes that each collision of the projectile in the target nucleus yields the same distribution of grey particles irrespective of the incident energy and that consecutive collisions contribute independently to the final $N_{g}$ distribution. This is true for light beams $B, C$ and $D$. But for the heavy projectile ${ }^{197} \mathrm{Au}$ although distribution shape is the same but its values are higher excepting the last point where it slightly decreases. For all the light beams $\left\langle N_{g}\right\rangle$ values increase and they have almost saturated for most central interactions [7].

(vii) $\left\langle N_{s}>/ N_{p a r t}\right.$ vs $\left\langle Q_{Z D}>/ Z_{\text {beam }}\right.$

The effect of nuclear geometry is further observed in the distribution of $\left\langle N_{s}\right\rangle / N_{\text {part }}$ when plotted against $\left\langle Q_{Z D}\right\rangle / Z_{\text {beam }}$, where $Q_{Z D}$ is the zero degree charge determined from the charge of PFs of $Z \geq 1$. Here again we stress that the parameter $Q_{Z D}$ can not be effectively used unless the charges of all the PFs are determined carefully especially in a heavy beam like
${ }^{197} \mathrm{Au}$ nuclei. The distribution of the high energy ${ }^{16} \mathrm{O}$ and ${ }^{32} \mathrm{~S}$ beams is quite similar and the same is the behavior between low energy beams of ${ }^{28} \mathrm{Si}$ and ${ }^{197} \mathrm{Au}$ but the magnitudes of high energy beams are much larger than the low energy beams as is depicted in Fig. 3(c). For the heavier beam, $\left\langle N_{s}\right\rangle / N_{\text {part }}$ is almost constant over the whole $Q_{Z D}$ range.

(viii) Global characteristics observed from the ${ }^{197}$ Au beam

We determined the maximum number of particles in each event by scanning with a fixed pseudorapidity window $\Delta \eta=0.2$ units across its full $\eta$ range. The average of this quantity $\left\langle\rho_{\max }\right\rangle$ is then plotted as a function of $N_{s}$ for all the beams. The least squares fit to the data is of the form $A+B N_{s}$, the values of $A=10.35 \pm 1.81$ and $B=0.41 \pm 0.01$. Our data in Fig. 4(a) show that the maximum number of particles in a given pseudorapidity interval increases linearly with multiplicity. This maximum number of particles demonstrates scaling [3] which can be very useful for future studies.

\section{(ix) $\left(d N_{s} / d \eta\right)_{p e a k}$ vs $<\eta_{l a b}>$}

Fig. 4(b) shows the maximum density as a function of $\left\langle\eta_{l a b}\right\rangle$. For each beam we observe that (a) the shift in $\Delta \eta$ is very small as function of density, (b) the shift in $\Delta \eta$ is always toward target side, due to more stopping and (c) the highest density is shown by the hezriest projectile ${ }^{197} \mathrm{Au}$ beam followed by the ${ }^{32} \mathrm{~S}$ beam. The contributions to $\left(d N_{s} / d \eta\right)_{\text {peak }}$ from the ${ }^{16} \mathrm{O}$ and ${ }^{28} \mathrm{Si}$ beams are comparable, though large differences in their energies is partially compensated by the ${ }^{28} \mathrm{Si}$ beam's heavier mass as compared to ${ }^{16} \mathrm{O}$ beam. This was also noted in Section (vii) Fig. 3(c).

(x) $\left(d N_{s} / d \eta\right)_{p e a k}$ vs $\left\langle N_{p}>/ Z_{\text {beam }}\right.$

In Fig. 4(c) we depict the maxima of the particle density distribution as a function of $\left\langle N_{p}\right\rangle / Z_{\text {beam }}$. A larger projectile would not only provide a larger interaction volume but also a somewhat larger energy density. Earlier in part (i) above, it was stated that the $\eta_{\text {peak }}$ shifts towards smaller $\eta$ values with increasing centrality. The width $\sigma_{0}$ also decreases, which is expected as the degree of stopping increases due to the increasing number of interacting participants from the target. The ratio of target participants to projectile participants increases with the decrease in the impact parameter. The values of $\left(d N_{s} / d \eta\right)_{\text {peak }}$ first increase linearly with centrality upto a value of $\left\langle N_{p}\right\rangle / Z_{\text {beam }} \approx 0.9$ for beams $B, C$ and $D$ except the last two points and for beam A, linearity is only upto 0.6 [Fig. 2(d)] but for the last 
two points the values are very large where the width $\sigma_{0}$ values decrease much more with centrality (Fig. 2b).

(xi) $P\left(\rho_{\max }\right)$

In order to find the probabilty of observing a given maximum particle density $\rho_{\max }$, for three beams ${ }^{28} \mathrm{Si}$, ${ }^{32} \mathrm{~S}$ and ${ }^{197} \mathrm{Au}$ using a scanning $\Delta \eta$ interval of 0.05 , we exhibit in Fig. 4(d) the energy density distribution of $\approx 4 \mathrm{GeV} /(\mathrm{fm})^{3}$ for the ${ }^{197} \mathrm{Au}$ beam which according to theoretical predictions should exhibit the presence of QGP. Perhaps we have to look for some other special signatures for the presence of QGP. Recently ${ }^{208} \mathrm{~Pb}$ on ${ }^{208} \mathrm{~Pb}$ experiment [16] have also observed in central collisions energy density $\approx 3 \mathrm{GeV} /(\mathrm{fm})^{3}$ but with no special signature of QGP.

\section{CONCLUSIONS}

For central collisions, the width is basically governed by the energy of the projectile. For centrality, $N_{p}$ is a good parameter for that one has to determine the charges of all the fragments of $Z>1$. The width parameter $\left(\sigma_{0}\right)$, the peak position $\left(\eta_{p e a k}\right)$ and centroid $\left(\left\langle\eta_{l a b}\right\rangle\right)$ of the pseudorapidity distributions decrease very slowly as a function of centrality for low energy beams ${ }^{28} \mathrm{Si}$ and ${ }^{197} \mathrm{Au}$ and are comparable to one and other but these values are relatively small when compared to high energy beams of ${ }^{16} \mathrm{O}$ and ${ }^{32} \mathrm{~S}$. Heavier projectile not only provide large interaction volume but also some what larger energy density. Shower particles of all the four beams show a scaling approximation in $\left\langle\rho_{\max }\right\rangle$ as a function of multiplicity. In ${ }^{197} \mathrm{Au}$ beam, an energy density of about $3-4 \mathrm{GeV} /(\mathrm{fm})^{3}$ is estimated for near head-on collisions with emulsion nuclei.

Acknowledgements

We are thankful to the technical staff and the emulsion operating groups of CERN and BNL for their help in the exposure and to Prof. G. Romano for the development of our emulsion stacks. This work was partially supported by the Department of Energy and partially by the Research Foundation of SUNY at Buffalo.

\section{References}

[1] K. Kajentie and L. McLerran, Ann. Rev. Nucl. Part. Sci. 37, 293 (1987) and references therein; Proceedings of the Q.M. 1990 Conference in Menton, France, Nucl. Phys. A 525 (1991).

[2] E. Karsch, Z. Phys. C 38, 147 (1988).
[3] P. L. Jain, A. Mukhopadhyay, G. Singh, Phys. Lett. B 294, 27 (1992); P. L. Jain, G. Singh and K. Sengupta, Z. Phys. C 52, 465 (1991); G. Singh, P. L. Jain and K. Sengupta, Phys. Rev. Lett. 61, 1073 (1988).

[4] P. L. Jain, K. Sengupta and G. Singh, Phys. Rev. C 44, 844 (1991).

[5] P. L. Jain, G. Singh and A. Mukhopadhyay, Phys. Rev. C 50, 1085 (1994) and references given therein.

[6] P. L. Jain and G. Singh, Nucl. Phys. A 586, 700 (1996); G. Singh and P. L. Jain, Phys. Rev. C 50, 2508 (1994).

[7] P. L. Jain, G. Singh and K. Sengupta, Phys. Rev. C43, R2027 (1991); K. Sengupta, G. Singh and P. L. Jain, Mod. Phys. Lett. 5, 285 (1990); ibid, 6, 29 (1991).

[8] T. Abbot et al., Phys. Rev. C 45, 2933 (1992); R. Albrecht, Z. Phys. C 55, 539 (1992).

[9] J. Barrette et al., Phys. Rev. C 46, 312 (1992); J. Barrette et al., Phys. Rev. Lett. 70, 2996 (1993).

[10] G. Singh, K. Sengupta and P. L. Jain, Phys. Rev. C41, 999 (1990); G. Singh and P. L. Jain, Z. Phys. A 344, 73 (1992).

[11] P. L. Jain, G. Singh and A. Mukhopadhyay, Phys. Rev. Lett. 74, 1534 (1995); P. L. Jain and G. Singh, Phys. Lett. B 366, 32 (1996) and references given therein.

[12] P. L. Jain, M. Kazuno and B. Girard, Phys. Rev. Lett. 32, 1460 (1974); P. L. Jain, M. Kazuno, B. Girard and Z. Ahmad, Phys. Rev. Lett. 32, 797 (1974).

[13] M. I. Adamovich et al., Nucl. Phys. A 593, 535 (1995).

[14] T. Akesson et al., Nucl. Phys. B 333, 48 (1990); ibid, Nucl. Phys. B 353, 1 (1991).

[15] P. L. Jain, K. Sengupta and G. Singh, Nuovo Cim. A 99, 9 (1988).

[16] T. Alber et al., Phys. Rev. Lett. 75, 3814 (1995).

Figure Captions 
Fig. 1 (a) $\left(d N_{s} / d \eta_{l a b}\right) / N_{e v}$ vs $\eta_{l a b}$ for ${ }^{197} \mathrm{Au}$ at $10.6 \mathrm{~A}$ $\mathrm{GeV}$. (b) The same as (a) but for four beams ${ }^{16} \mathrm{O}$ and ${ }^{32} \mathrm{~S}$ at $200 \mathrm{~A} \mathrm{GeV},{ }^{28} \mathrm{Si}$ at $14.5 \mathrm{~A} \mathrm{GeV}$ and ${ }^{197} \mathrm{Au}$ at $10.6 \mathrm{~A} \mathrm{GeV}$.

Fig. 2 For four beams: (a) $\left\langle\eta_{l a b}\right\rangle$, (b) $\sigma_{0}$, (c) $\eta_{\text {peak }}$ and (d) $\left\langle N_{s}\right\rangle$ as a function of $\left\langle N_{p}\right\rangle / Z_{\text {beam }}$.

Fig. 3 (a) $\left\langle N_{\text {part }}>\right.$ and (b) $\left\langle N_{g}>\right.$ as a function of $\left\langle N_{p}\right\rangle / Z_{\text {beam }}$. (c) $\left\langle N_{s}\right\rangle /\left\langle N_{\text {part }}\right\rangle$ vs $\left\langle Q_{Z D}\right\rangle / Z_{\text {beam }}$.

Fig. 4 (a) $<\rho_{\max }>$ vs $N_{s}$, (b) $\left(d N_{s} / d \eta\right)_{\text {peak }}$ vs $<$ $\eta_{l a b}>$, (c) $\left(d N_{s}>/ d \eta\right)_{p e a k}$ vs $\left\langle N_{p}>/ Z_{\text {beam }}\right.$ and (d) $P\left(\rho_{\max }\right)$ vs $\rho_{\max }$.
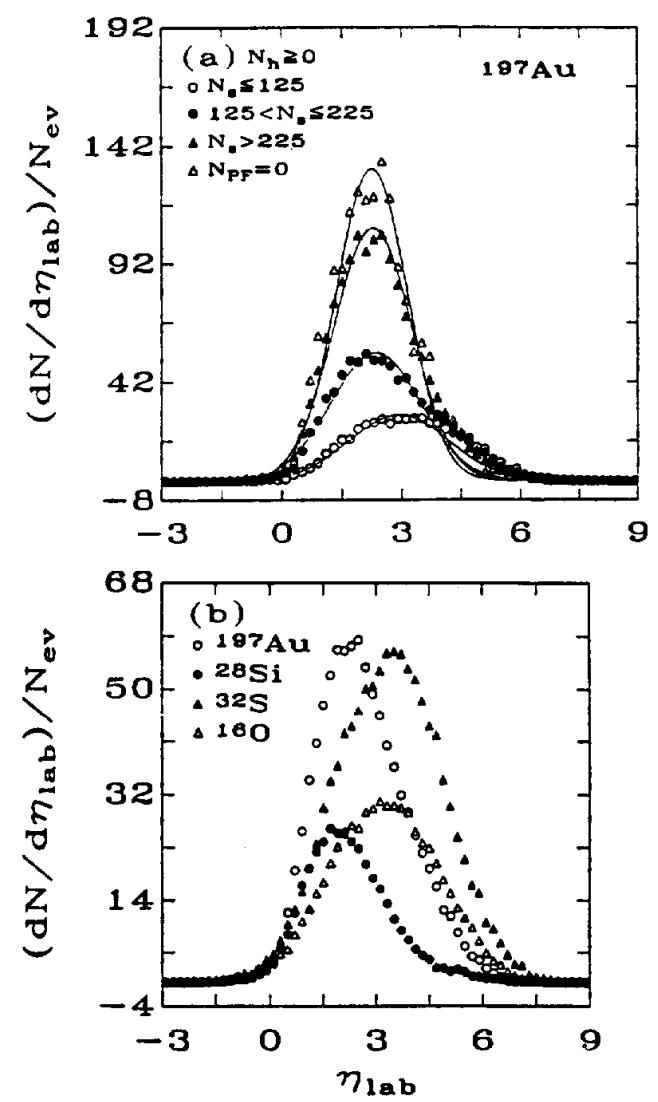
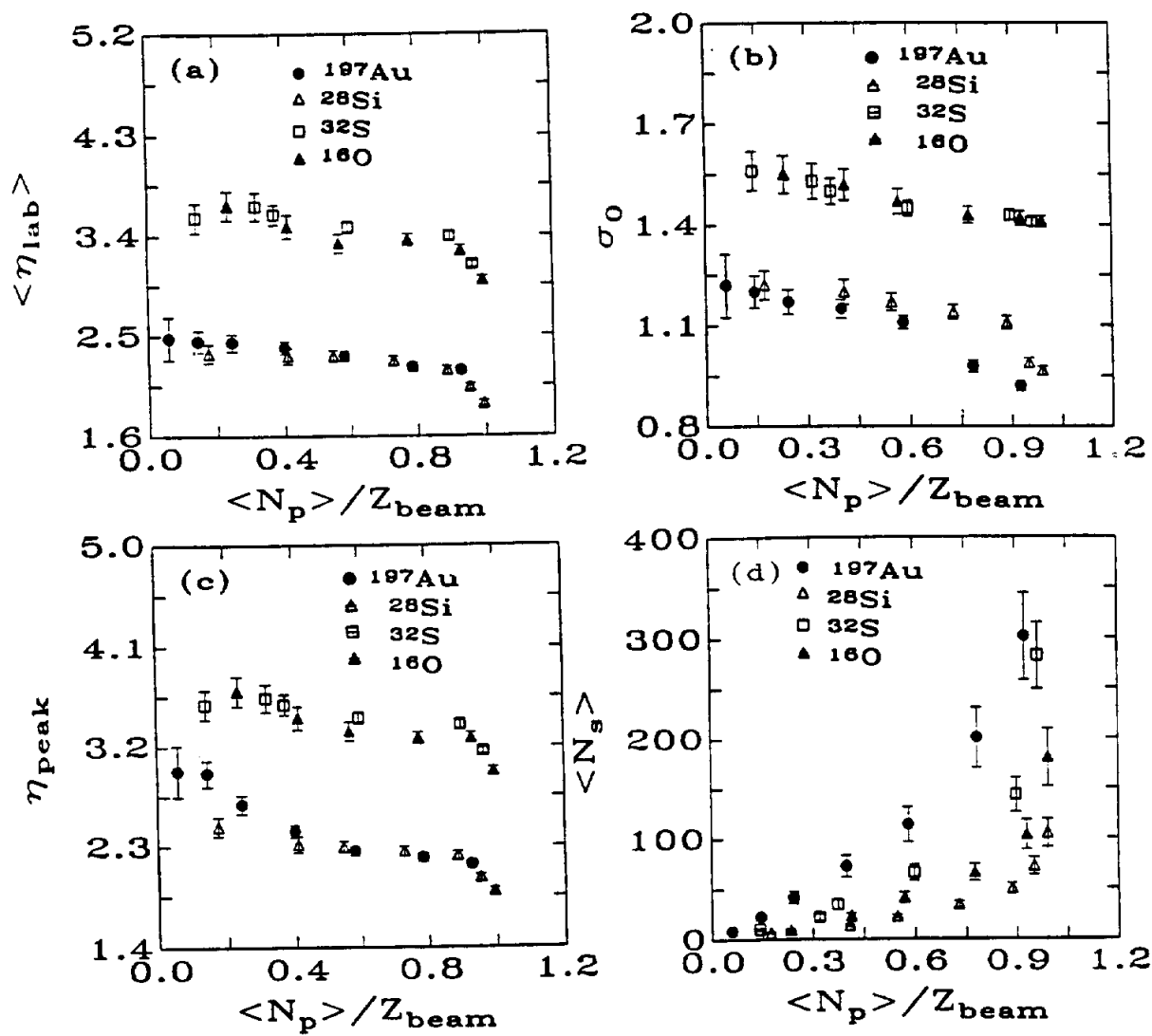

Fig. 2 

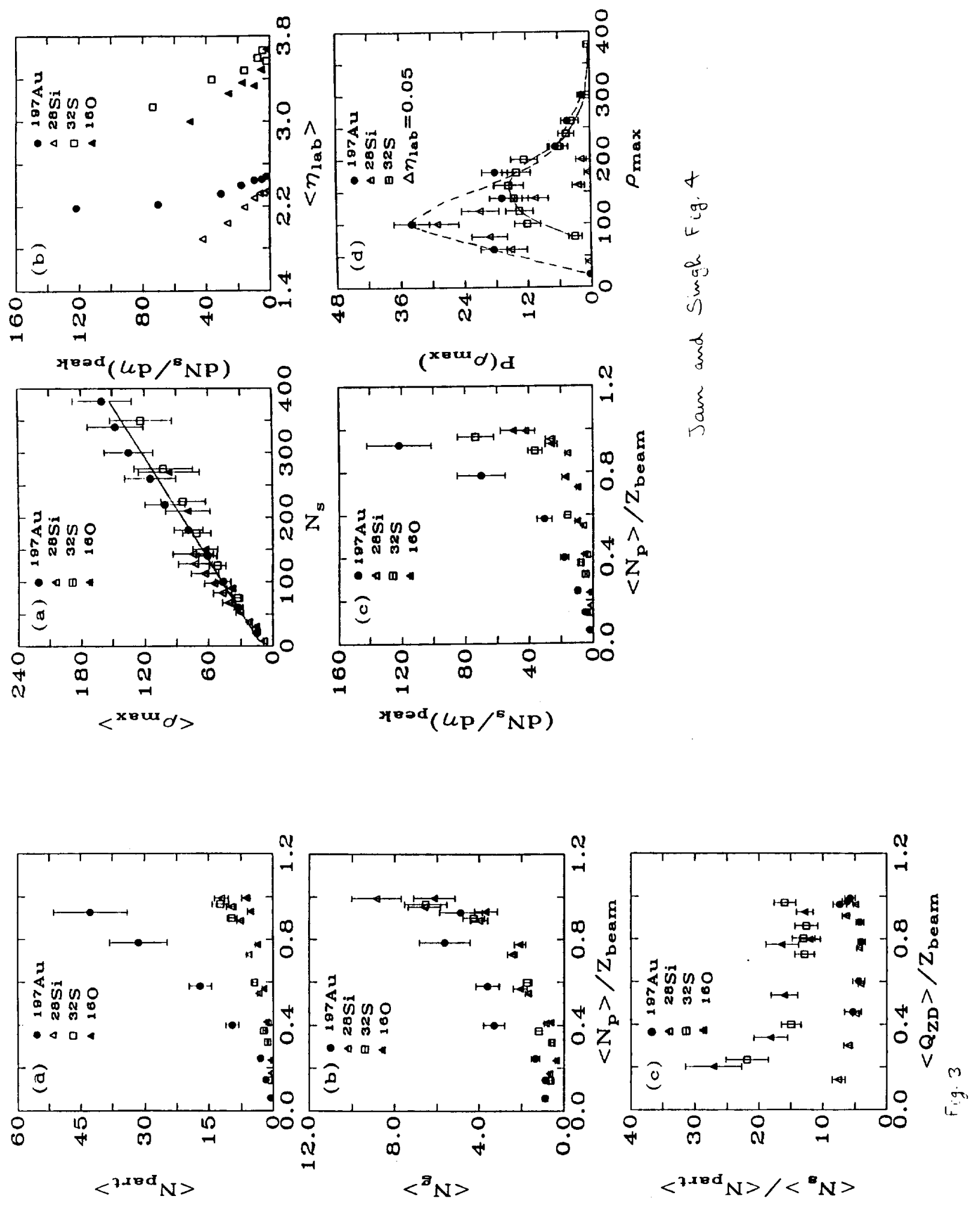
